\title{
Damage to pharynx and esophagus due to bone soup
}

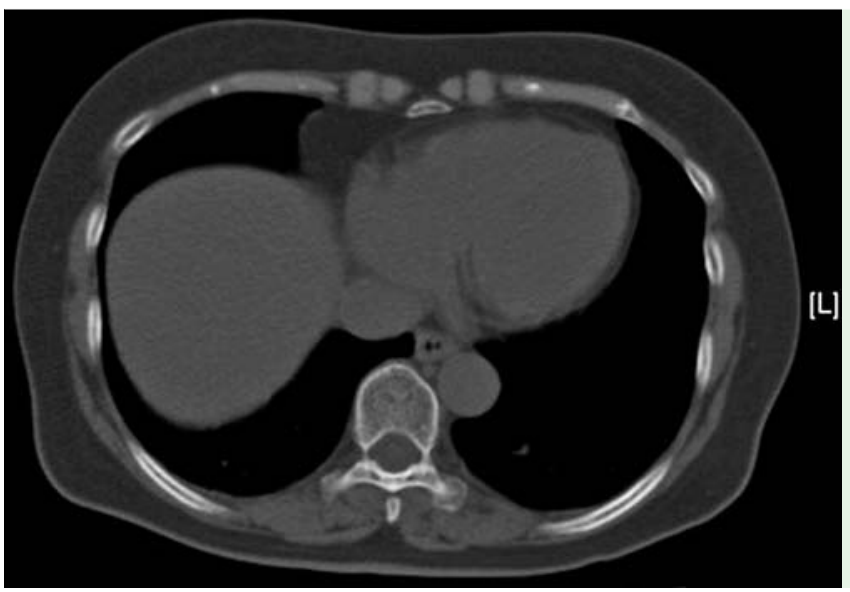

Fig. 1 Computed tomography (CT) scan showing diffuse wall thickening in the upper and middle esophagus.
A 61-year-old woman sought medical attention after experiencing throat irritation, chest pain, and difficulty swallowing for 1 week. Chest computed tomography (CT) showed diffuse wall thickening in the upper and middle esophagus, as well as an esophageal stricture ( Fig.1), suggestive of esophageal carcinoma. An esophagogastroduodenoscopy (EGD) was performed. Before the endoscopy, a slightly tender swelling was observed on the right side of the neck. The endoscopy revealed swelling in the left side of the pharynx ( $\bullet$ Fig. 2a) and at the entrance to the esophagus ( Fig. $\mathbf{2}$ b), and a stenosis was also apparent; however, the lumen and mucosa exhibited normal elasticity and the lumen distended normally after air inflation. Some regions of the esophageal mucosa appeared purplish-blue ( $\bullet$ Fig. 2c), although the mucosal surface was smooth with no abnormalities.
The patient was given a semi-liquid diet and mucosa-protecting treatments, including esomeprazole, magnesium hydroxide, enteric-coated tablets, and Talcid. Her symptoms, including the difficulty swallowing and chest pain, gradually improved. A repeat upper gastrointestinal endoscopy performed 6 weeks later showed that the pharyngeal swelling had disappeared ( Fig.3), the esophageal mucosa had become pink, and there were no unstained areas observed after dyeing with Lugol dye. An endoscopic ultrasound (EUS) clearly showed the esophageal mucosal layer, within which no abnormal thickening or other abnormalities were observed ( $\bullet$ Fig. 4 ).

The patient's medical history indicated that she had ingested chicken bone soup containing very small but sharp bones 2 days before her symptoms appeared. She had immediately experienced throat dis-

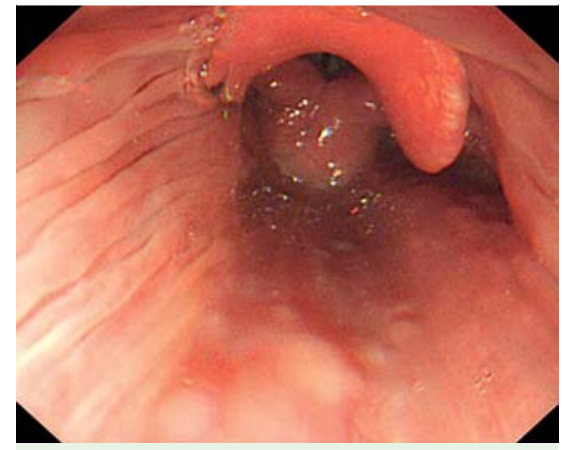

Fig. 3 Repeat endoscopy 6 weeks later showing that the pharyngeal swelling had disappeared.

comfort but had ignored this. Damage to the pharynx and esophagus by foreign bodies usually manifests as a mucosal injury; however, this patient exhibited mainly pharyngeal submucosal swelling, diffuse esophageal submucosal edema, and submucosal congestion. We believe this is because the tiny scars in the mucosa damaged by the small sharp bones had healed up by the time of endoscopy, so that no damage was found on the mucosal surface although there were still submucosal hematomas. Therefore, in addition to endoscopic diagnosis, evaluation of the medical history and follow-up observations were required in order to distinguish between melanoma of the esophagus and diffuse esophageal spasm.

\section{Endoscopy_UCTN_Code_CCL_1AB_2AC}

\section{Competing interests: None}

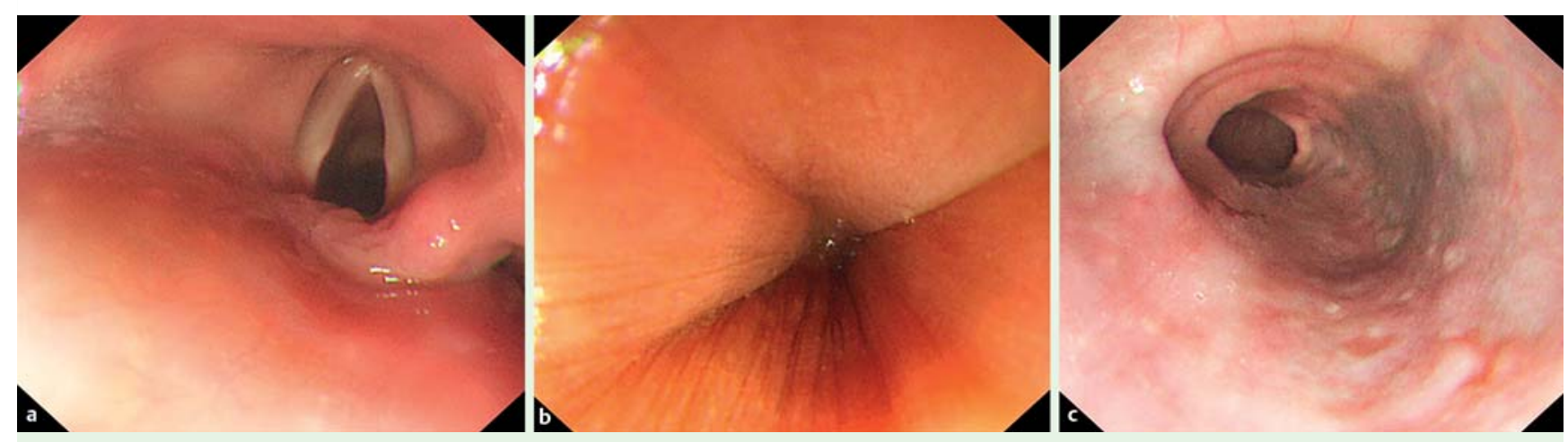

Fig. 2 Endoscopic views showing: a swelling of the left side of the pharynx; $\mathbf{b}$ the entrance to the esophagus; $\mathbf{c}$ the esophageal mucosa, which in places appeared purplish-blue. 


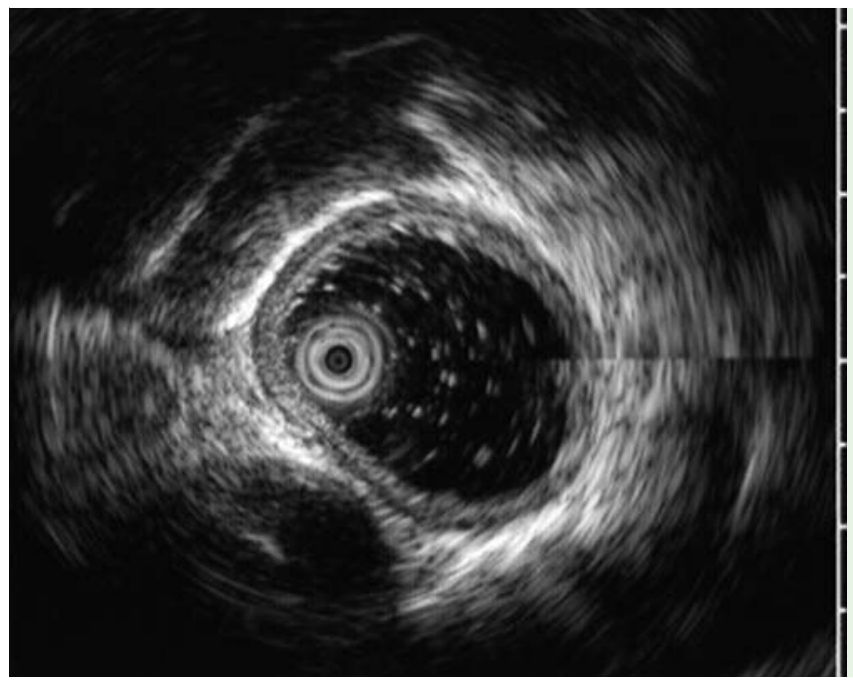

Fig. 4 Endoscopic ultrasound (EUS) showing a normal appearance of the esophageal mucosal layer.

\section{Xiao-jun Zhao, Xin Wang, Ai-qin Li,} Jian-qiu Sheng

Department of Gastroenterology, Beijing Military General Hospital, Beijing, China

Bibliography

DOI http://dx.doi.org/

10.1055/s-0034-1377206

Endoscopy 2014; 46: E324-E325

(c) Georg Thieme Verlag KG

Stuttgart · New York

ISSN 0013-726X

\section{Corresponding author}

Jian-qiu Sheng, MD

Department of Gastroenterology

Beijing Military General Hospital

Nanmenchang 5\#

Dongcheng District

Beijing 100700

China

zhaoxiaojun2@163.com 